\title{
Transcription goes digital
}

\author{
Timothée Lionnet ${ }^{1,2} \&$ Robert H. Singer ${ }^{1,2+}$ \\ ${ }^{1}$ Department of Anatomy \& Structural Biology, and ${ }^{2}$ Gruss Lipper Biophotonics Center, Albert Einstein College of Medicine, \\ Bronx, NY, USA
}

Transcription is a complex process that integrates the state of the cell and its environment to generate adequate responses for cell fitness and survival. Recent microscopy experiments have been able to monitor transcription from single genes in individual cells. These observations have revealed two striking features: transcriptional activity can vary markedly from one cell to another, and is subject to large changes over time, sometimes within minutes. How the chromatin structure, transcription machinery assembly and signalling networks generate such patterns is still unclear. In this review, we present the techniques used to investigate transcription from single genes, introduce quantitative modelling tools, and discuss transcription mechanisms and their implications for gene expression regulation.

Keywords: imaging; bursting; chromatin; gene expression noise EMBO reports (2012) 13, 313-321; published online 13 March 2012;

doi:10.1038/embor.2012.31

See Glossary for abbreviations used in this article.

\section{Introduction}

Cellular phenotypes result from the expression of many genes, organized in networks. Correct gene expression is required to ensure that cells survive in their environment and make the correct decisions [1]. The process of expressing a gene begins with transcription, which implies that any fluctuation at the level of mRNA synthesis has the potential to propagate to the downstream protein pool. Transcriptional control is therefore a crucial cellular mechanism to avoid over- or underexpression of genes. Despite continued progress that has identified many of the actors of transcription and their whereabouts in the genome-components of the transcription machinery, transcription factors and chromatin remodellers-it is still often not possible to tell in which order, how often and how regularly the various events leading to transcription occur. The molecular interactions at the promoter are still too fast to allow for intermediates to be 'caught' by using biochemical methods.

In this context, recent progress to image transcription in live cells has provided invaluable new insights [2] into the behaviour and function of specific actors over timescales ranging from seconds to

\footnotetext{
${ }^{1}$ Department of Anatomy \& Structural Biology, Albert Einstein College of Medicine, Bronx, NY 10461, USA

${ }^{2}$ Gruss Lipper Biophotonics Center, Albert Einstein College of Medicine, Bronx, NY 10461, USA

+Corresponding author. E-mail: robert.singer@einstein.yu.edu
}

Received 27 December 2011; accepted 23 February 2012; published online 13 March 2012 days. Imaging the process in live cells and in real time has proven to be a powerful tool for the quantitative testing of the various models of gene expression regulation [3]. It has also brought a surprise: what we thought was a tightly regulated, deterministic process is actually very heterogeneous within a cell population and highly variable over time. Single-cell experiments have allowed the quantification of these two variables and have uncovered new modes of regulation: transcription can occur through isolated pulses [4], with the corollary that cells can deal with signals in a digital rather than analogue way.

Here, we review recent progress made in characterizing transcription dynamics using single-cell experiments. We present the evidence for transcriptional 'bursting' and the theoretical tools by which it is quantified, and discuss the biological mechanisms that could generate such signatures. Finally, we examine how, when and why cells have adopted digital modes of gene expression.

\section{Monitoring transcription from single genes}

Various techniques have been developed to image transcription at the single-cell level. The first demonstration of single-molecule mRNA detection in cells occurred more than a decade ago by using mRNA fluorescence in situ hybridization (mRNA FISH; [5]). By using multiple fluorescent DNA probes binding to a given mRNA in conjunction with highly sensitive and spatially precise microscopy, it is possible to detect single molecules of mRNA. This powerful technique can interrogate any gene of interest without the need for a reporter system, and has now been applied in a variety of systems [6-10]. Although it is limited to fixed cells, various mathematical models have been used to infer dynamics from the distribution of mRNA expression over a cell population. In living cells, the most widely used system to directly observe single mRNA molecules is the MS2 system [11]. This system exploits the high affinity of MCP for a short RNA sequence contained in the bacteriophage genome. It requires that the gene of interest is modified to contain multiple copies of the sequence (MBS) and that cells express MCP fused to a fluorescent protein such as GFP. As the RNA is expressed, the newly formed MBS hairpin is bound tightly by the fluorescent MCP-GFP fusion. This process has been used extensively to study transcription in various systems [12-17]. Originally used in higher eukaryotes in the context of gene arrays, where many $(100-1,000)$ copies of the reporter are inserted at a given locus, the method has recently been extended to a single-gene copy [9], and even as an endogenous gene in a knock-in mouse [12], proving its broad potential and limited disruptiveness. The binding of the fluorescent protein to the nascent chain occurs much faster than the timescales of seconds involved in typical transcription dynamics [13]. The principle of a 


$\begin{array}{ll}\text { Glossary } & \\ \text { GFP } & \text { green fluorescent protein } \\ \text { MBS } & \text { MS2 binding site } \\ \text { MCP } & \text { MS2 coat protein } \\ \text { MS2 } & \text { bacteriophage MS2 } \\ \text { NF-kB } & \text { nuclear factor kB } \\ \text { PP7 } & \text { Pseudomonas phage 7 } \\ \text { Pol II } & \text { RNA polymerase II }\end{array}$

high-affinity fluorescent tag binding to a specific mRNA sequence has been used in various proposed reporter systems [18], but apart from MS2, single-molecule resolution has only been demonstrated in the case of PP7, an homologous system [13]. Apart from directly imaging mRNA, other techniques use mathematical modelling based on the fluorescent [19-23] or luminescent [24,25] signal from a protein reporter to infer the dynamics of the mRNA intermediate. The reporters can be destabilized so that the fluorescence or luminescence readout at a given time only comes from recently synthesized proteins. The mathematical methods used to reconstruct the transcriptional activity based on the time variation of the protein fluorescence range from the simple-when looking at transient induction $[19,21]$ or rare transcriptional events $[20,22,23]$ — to the complex, in the case of steady-state bursting [24,25]. Proteinbased techniques present the advantage of signal amplificationone mRNA usually gives rise to many proteins, making detection easier-but are limited in resolution, as they cannot resolve events faster than the timespan between transcription and translation of the mRNA (typically minutes) and the lifetimes of the proteins.

\section{Transcription occurs continuously or in bursts}

The variety of systems studied — from bacteria to human cells-has revealed an impressive spectrum of transcription dynamics, from continuous to pulsing, from synchronized to uncorrelated. The main surprise of the past years has been the discovery that transcription can be highly discontinuous, occurring through bursts of activity (gene 'on'), in which many mRNAs are transcribed in a short time, interspersed with periods of inactivity (gene 'off'). Originally suggested as an explanation for the observed variations in protein levels over a cell population [26], the first direct observation of transcriptional bursting was made in bacteria with a reporter gene [17]. It seems to constitute a ubiquitous mode of transcription, as it has also been observed in slime mould [16], fly [27], rat [24], mouse [24,25] and human [28] cells, in both endogenous genes and artificial constructs (Table 1). mRNA FISH experiments in fixed metazoan tissue have also suggested the existence of transcription pulses [27,29]. Bursting is not restricted to Pol II in eukaryotes: Pol I transcription of ribosomal genes also occurs through bursts $[30,31]$. The duration of the bursts and the interval separating the bursts vary from a few minutes to many hours. Work in bacteria has shown that the 'burstiness' of a gene could be predicted based only on the mean expression level, suggesting that bursting is a gene-independent process [8]. In this study, higher expression led to higher 'burstiness'. The observation of a global bursting mechanism is universal: in yeast, noise scales with the expression level in a gene-independent fashion [32]; in mice, many observed genes display the same features of bursting despite important variation in their kinetics [25]. Transcription bursts can be observed in response to a given signal, but also occur in the absence of any signal $[24,25]$. Whereas well-timed bursts can be observed following transcription induction $[19,20,33]$, steady-state bursts are usually random in their timing $[16,17,24,25]$.

Despite the observation of transcription bursts in many systems, it is not the sole expression mode. Genes in bacteria $[22,23,34]$, yeast [10,13] and human [9] cells can exhibit a constitutive transcription signature in which transcription events occur randomly over time, with a constant probability. Constitutive gene expression is prevalent among housekeeping genes [35] and consistent with a model in which the gene is continuously 'on'. Single events of transcription factor binding to the promoter initiate transcription of a single message, suggesting a 'hit-and-run' model [13]. Transcriptional initiations involve only the gene locus as distinct genes, and even two copies of the same gene initiate transcription independently [36].

\section{Two burst types: spontaneous compared with transmitted}

Considerable theoretical effort has been devoted to developing quantitative models of transcription [37-39]. Conceptually, two non-mutually exclusive classes of mechanism can give rise to discontinuous transcription (Fig 1A). The first consists of pulses that merely propagate changes in upstream signalling (extrinsic bursting): for example, transiently high concentrations of activator induce a burst of transcription before the gene shuts off such as in regulatory networks consisting of multiple feedback loops [21,40]. The second class of mechanism, termed intrinsic or spontaneous bursting, relates to genes that spontaneously switch between their 'off' and 'on' state. Once the gene is 'on', multiple transcripts are generated before turning off. Stochasticity in transitions between the two states is the sole driving force of the 'bursty' behaviour, so bursts can occur intrinsically, in the absence of any genetic regulation or external signalling. The contributions of extrinsic and intrinsic factors to the observed bursts are difficult to deconvolve experimentally [41], but have crucial implications for the regulation of gene expression. A spontaneously bursting gene generates a more variable expression profile across a cell population than a gene that faithfully transmits the fluctuations in its environment: the concentration of a given hormone, for example. Note that from a mathematical point of view, bursting is also observed if the gene displays memory-that is, if a short interval between initiation events tends to be followed by another short interval [42].

Extrinsic bursting has been demonstrated in various systems $[19-21,33,43]$. Depending on the architecture of the genetic network driving the observed gene, the timing of the pulses can vary from completely stochastic [21] to very precise [19]. As all direct targets of the same regulator are sensitive to its concentration, their expression is correlated at the single-cell level.

Spontaneous bursting is difficult to determine unambiguously [41], but its existence has been clearly established when two alleles of a given gene in the same cell were observed to burst independently [24]. Although it is unclear whether all bursting housekeeping genes fall into the intrinsic category, it is a common assumption in the field. Despite this caveat, the accumulated biological evidence for intrinsic bursting suggests a broad, gene-independent mechanism, occurring in both eukaryotes and prokaryotes.

\section{A quantitative model of stochastic bursting}

A theoretical model, termed the 'random telegraph', has been successful at providing a unified description of stochastic bursting, regardless of its origin [44]. In this model, a gene randomly switches between periods in which it is permissive for transcription ('on') and 
Table 1 | Compiled live-cell evidence for transcription bursts

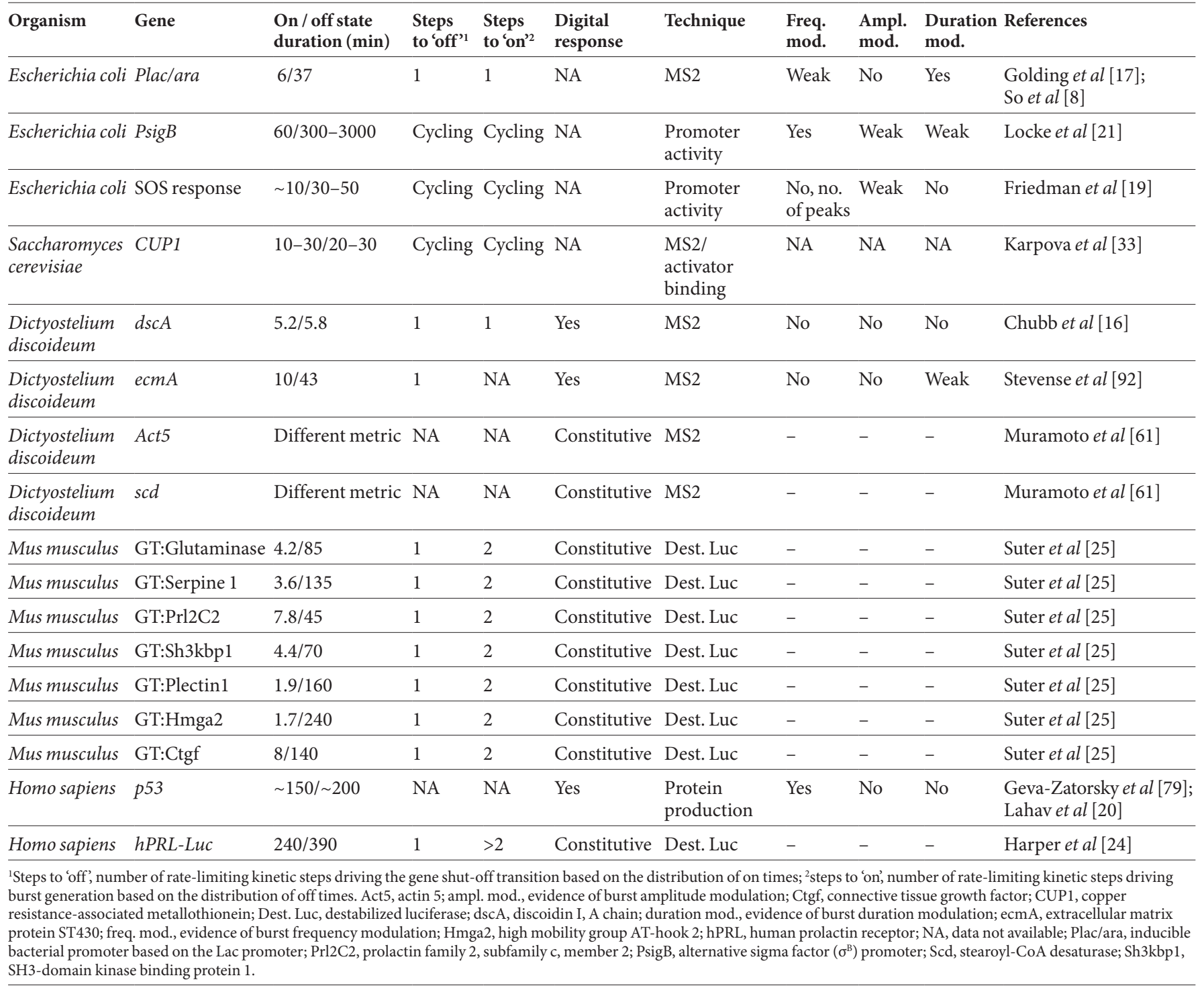

periods of inactivity ('off'). When the gene is 'on', transcription events fire randomly over time. This model is attractive because it captures a vast range of transcriptional dynamics within a single mathematical framework: 'bursty' transcription corresponds to infrequent transitions to the permissive state, but the permissive gene initiates transcription at a high frequency; on the other hand, constitutive expression can also be described within the same scheme-gene continuously 'on', that is, very infrequent transitions to 'off'. Another appealing aspect of the model is its generality: there is no assumption made as to what event drives the transitions between inactive and active states; therefore the model can describe both spontaneous bursting and stochastic bursting in response to a noisy upstream signal-for example, random fluctuations in the concentration of a transcription factor generate intermittent bursts of activity. Importantly, analytical solutions to the model exist and can be used to determine the various kinetic rates from the distribution of the mRNA copy number across a cell population [6,10]: 'bursty' genes tend to produce broader profiles of expression across a cell population than constitutively expressed ones. Note that caution must be applied to distinguish bursting from other sources of variability in such analyses; different sources of variability such as partitioning of mRNA during cell division can give rise to similar copy number distributions [45]. Despite this caveat, it is possible to use the model as a common metric for all genes studied. As the model explicitly assumes that single random events drive the transitions from the two states, it follows that both the duration of the bursts and the time between bursts should be exponentially distributed. This assumption of the model holds well in the case of genes shutting off: all burst durations observed so far are exponentially distributed $[16,17,24,25,46]$. This observation indicates that the time a burst lasts is determined by a single, rate-limiting event. In the case of the duration of the 'off' state, recent experiments in mammalian cells have suggested that each burst is followed by a refractory period lasting up to $3 \mathrm{~h}$, during which a new burst cannot take place $[24,25]$. This result implies that multiple sequential steps with similar rates are required to switch a gene 'on', a situation that is not yet well-described by the model. 


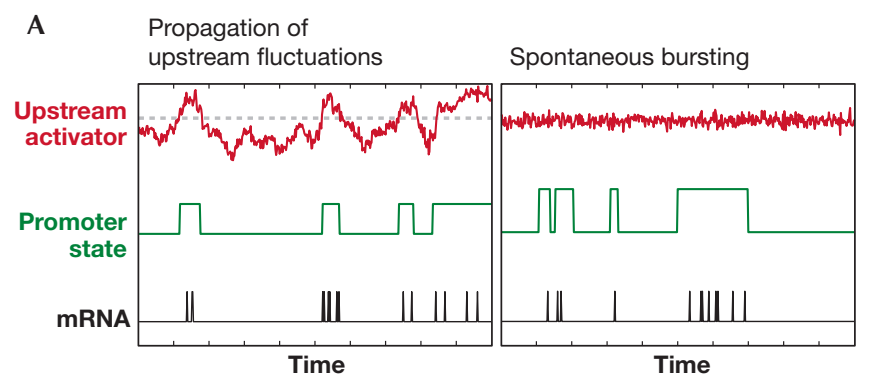

B
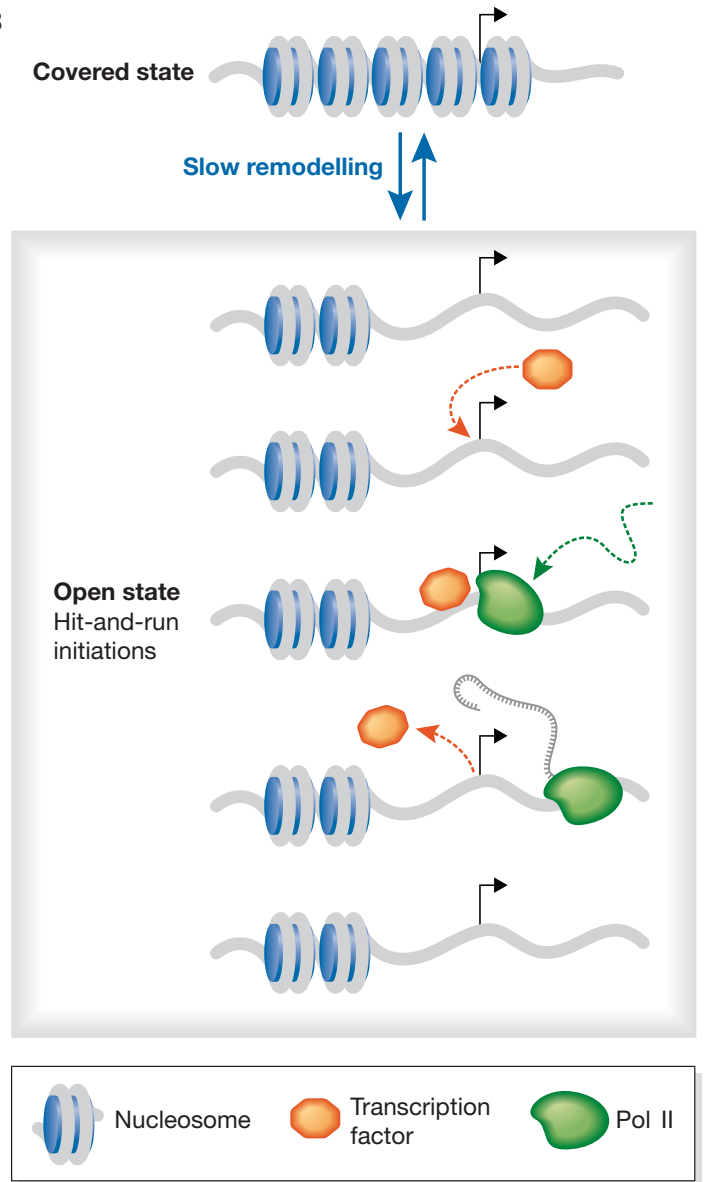

Fig 1 | Models of transcription bursts. (A) Left: bursts can appear when fluctuations in the concentration of an upstream factor reach a threshold (grey dashed line), triggering the 'on' state of the promoter, allowing transcription to take place. Right: spontaneous bursts occur even when upstream signalling is constant. Red: concentration of an upstream activator. Green: state of the promoter; 'off' corresponds to the baseline, 'on' corresponds to the high level. Black: each vertical line represents the initiation of the transcription of a single mRNA. (B) Chromatin-based model of bursting: a slow step corresponding to nucleosome dissociation or histone mark deposition triggers the switch from a covered promoter state (off) to an open promoter state (on). In the open state, many sequential initiations are made possible by transient interactions of transcription factors with the promoter.

\section{The origin of bursting remains mysterious}

Despite its success in establishing a universal description of transcription dynamics, the random telegraph model remains essentially phenomenological as it does not explain what biological steps underlie its kinetic parameters. Bursting has been observed ubiquitously, suggesting a common underlying mechanism. However, such a mechanism has so far been elusive. The timescales involved in bursting vary by two orders of magnitude over the range of systems studied: from a few minutes in bacteria [17] and lower eukaryotes [16] to a few hours in higher eukaryotes [24,25]. Refractory periods have not been observed in prokaryotic cells, and they are not always present in eukaryotic bursts [16]. The refractory period might involve slow transcriptional processes, as shorter bursts (minutes) did not feature such periods, in both a prokaryotic [17] and a eukaryotic context [16]. This wide variety of dynamic behaviours might indicate that there are multiple biological mechanisms leading to transcription bursts.

To function, the transcriptional machinery requires the formation of a complex of dozens of proteins at a gene's promoter $[47,48]$. Because the assembly of such a large multi-subunit complex is an energetically demanding process, it would seem natural that, once formed, it would be sufficiently stable to allow the synthesis of multiple mRNAs. This picture, inherited from biochemical studies, would explain bursting through the rare but stable formation of transcriptional complexes that allow efficient 're-initiation'. However, the stability of complexes at the promoter has recently been challenged by various imaging studies showing that residence times rarely last more than minutes or even seconds [49]. Clearly, more effort is needed to reconcile conflicting results obtained from imaging and biochemistry, but the transient nature of interactions at the promoter hints at other potential mechanisms for bursting.

Ever since the demonstration that chromatin disruption increases the variability of expression of a 'bursty' reporter gene in yeast [50], chromatin has been suggested as a determinant of transcriptional bursting. Genome-wide mapping of nucleosome occupancy has shown that promoters are usually depleted of nucleosomes upstream from the transcription start site (TSS; [51]). Commonly, highly active genes correlate with lower nucleosome occupancy, consistent with more frequent transcription factor binding and pre-initiation complex formation. Recent bioinformatics studies have refined this picture and have shown that genes can be divided into two groups based on the nucleosome occupancy levels at their promoters [52-54]: open promoters have a well-defined nucleosome-depleted region proximal to the TSS, due to the presence of DNA sequences that destabilize nucleosome binding. On the other end, covered promoters feature high nucleosome occupancy immediately upstream from the TSS, with the corollary that transcription factors have to compete with nucleosome binding at the promoter. The open promoters are typically found upstream from essential genes and correlate with lower expression variability. Interestingly, the covered promoters are enriched in non-essential genes and genes activated in response to signalling. Covered promoters correlate with higher histone turnover, higher TATA box frequency and higher expression variability. Single-mRNA counting analyses of transcriptional noise in yeast confirm the qualitative difference in expression noise between gene types: housekeeping genes display a robust, constitutive expression, whereas cell-cycle-regulated and stress-response genes display a much higher variability, consistent with bursting [35]. Furthermore, genes that have more variable transcript copy numbers based on observations from single-mRNA counting experiments display a 
more pronounced nucleosome-depleted region upstream from the TSS (Fig 2). The competition between nucleosomes and transcriptionfactor-binding sites, and its effect on transcriptional dynamics, has been investigated recently in yeast [55]. Two cell-cycle-regulated genes that contain a well-defined nucleosome-depleted region in their promoter reliably produce a pulse of expression every cell cycle. In constructs where the promoter of each gene was modified so that a nucleosome occluded the binding site of the gene activator, the pulses still occurred at the right time in the cell cycle and had similar amplitude, but did not occur during every cell cycle. The all-or-nothing control of nucleosome occupancy on transcription is consistent with previous results showing that nucleosome occupancy affects the induction threshold of a gene, rather than its expression level [56]. A recent genome-wide analysis has further refined the distinction between the open and covered classes and also supports a model in which changes in the chromatin structure at the promoter drive the on-off transitions [57]. In such a model, slow (minutes or longer) changes in nucleosome occupancy or histone marks generate a permissive or repressive state for transcription. When the gene promoter is open, transcription factors and components of the transcription machinery turn over fast—seconds or less [58]—at the gene locus, enabling multiple rounds of initiation, potentially through a hit-and-run model in which each complex formation leads to the synthesis of a single mRNA (Fig 1B) or some variation such as the transcriptional ratchet [59]. This model is consistent with biochemical observations of cyclic changes in the promoter state over a few hours after transcription induction [60]: transcription factors, histones, chromatin remodellers and components of the transcription machinery display well-timed oscillations in their promoter-bound fraction when averaged over a cell population. The decoupling of these two timescales has been shown in copper response genes in yeast [33]: whereas the promoter-bound fraction of a transcriptional activator was found by using biochemical methods to cycle at a slow rate (30-40 min period), the activator was measured to turnover within 2 min at the gene locus in single cells. This apparent paradox can be explained if we assume that the promoter slowly switches between the open and covered states. Remarkably, the cycles of activator occupancy negatively correlated with cycles of histone occupancy at the promoter. As an additional line of evidence for the role of chromatin in bursting, the transcriptional pulsing frequency was shown to be inherited through mitosis in Dictyostelium discoideum in a mechanism dependent on H3K4 methylation [61].

Despite the attractiveness of the role of chromatin in shaping transcription bursts, there have to be other mechanisms: first, although bacteria lack higher-order chromatin, they nevertheless display transcription bursts [17]; second, marked changes in chromatin state or in chromosomal locus can have moderate effects on the bursting signature [25]. Other potential sources of bursting include local DNA topology effects, or DNA conformational changes at the gene that induce episodes of highly efficient transcription. Such local conformation changes could involve gene loops, in which terminating polymerases can immediately reengage in transcription [62]. Such loops have been implicated in various systems, including yeast, human and virus genes, by using chromosome conformation capture. The formation of efficient entry compartments in which Pol II is efficiently recycled after terminating transcription has also been observed in the absence of DNA looping in the fly heat-shock genes [63]. Finally, genes have been proposed to be actively expressed within transcription factories,
A

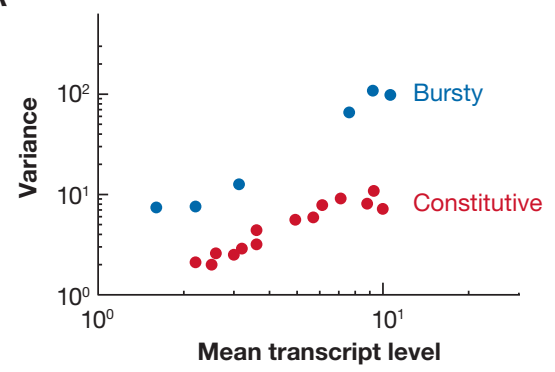

B

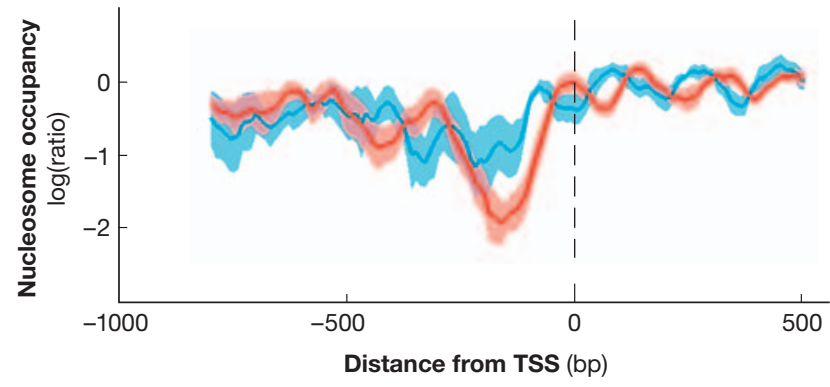

Fig 2 Relationship between transcriptional noise and promoter architecture in yeast genes. (A) Plot of the variance compared with mean mRNA copy number for various yeast genes studied by single-molecule FISH reveals two modes of expression: 'bursty' (high variance, blue) and constitutive (Poissonlimited variance, red). Housekeeping genes all fall in the constitutive class. All but one cell-cycle-regulated and signal response genes fall in the 'bursty' class. Data adapted from Lionnet et al [35]. (B) Promoters of 'bursty' genes have a covered architecture (blue), whereas constitutively expressed genes display a pronounced nucleosome-depleted region (red). Data from Lee et al [91], solid line for each class is the average of the genes from panel A, shaded area indicates mean \pm s.e.m. TSS, transcription start site.

where many associate locally to form a locus enriched in transcription machinery components [64]. All these models have a common point: they assume the formation of a local compartment in which transcription components are transiently highly concentrated, thereby increasing initiation frequency. This is the main difference from the chromatin model, in which the increase in transcription initiation occurs by a modulation of the access of factors to the promoter, not the local concentration of the factors. As these effects are not mutually exclusive, they could synergize.

\section{Transcription control beyond initiation}

Although initiation has long been thought to be the main regulatory step of transcription, recent data suggest that post-initiation steps can also have marked effects on transcription efficiency. High-throughput techniques have demonstrated that a considerable fraction of genes $(30 \%)$ feature an enrichment of Pol II in a paused state, immediately downstream from the promoter $[65,66]$. Imaging studies have suggested that the inefficiency of the progress of Pol II through promoter escape and pausing release could result in as little as $1 \%$ productive Pol II promoter-binding events [14]. Although pausing appears to be widespread, its biological role is still unclear. Paused genes are enriched for signal response genes and developmentally regulated genes [66], and feature low levels of nucleosome occupancy 
downstream from the TSS, in spite of sequences that would normally favour a covered architecture [67]. These findings suggest that promoter proximal pausing could maintain an open chromatin structure around the promoter. After the release of the paused polymerase upon signalling, multiple rounds of transcription would be facilitated by the permissive chromatin structure. According to this model, pausing could thus lead to transcriptional bursting. Another dynamic consequence of pausing is that it maintains the promoter in a state in which it is ready to start productive transcription. Paused genes might therefore respond faster to signalling. Studies of transcriptional activation during development have supported the idea that some genes are paused before their activation: the paused state is released only in specific tissues upon signalling, and the paused genes display a synchronous response at the tissue scale [68]. By contrast, nonpaused genes show a more variable activation profile. Despite recent progress, the effect of pausing on rapid induction is still unclear [69], and pausing might be involved in other regulatory mechanisms, such as the maintenance of local chromatin profiles [70], or allowing promoter-bound Pol II to coordinate gene expression through the recruitment of other factors [69].

Other regulatory checkpoints exist beyond initiation and release from pausing. Although such measurements have long been out of reach, there is now some evidence that elongation rates can be modulated within individual cells, for example, during the cell cycle in yeast [13]. In addition, whereas elongation rates have long been thought to lie in the $0.5-4 \mathrm{~kb} / \mathrm{min}$ range [71], a recent finding suggests that Pol II could transcribe a gene under the control of the HIV LTR promoter at over an order of magnitude faster [72]. Notably, inherent variability in the rates of successive elongating polymerases could result in 'traffic jams' along genes when a leading slow polymerase blocks faster ones. Such situations could generate bursting without the need to invoke modulations in the initiation frequency; interactions between elongating polymerase complexes also have the potential to attenuate 'bursty' initiation profiles [73]. Elongation rate might therefore be an underappreciated regulatory lever, as it can shape the transcription profile. Elongation has also been suggested to affect Pol II processivity [74] and mRNA processing steps such as alternative splicing [75]. Finally, single-cell studies of transcription suggest a rate-limiting step of approximately 1 min at the end of elongation $[10,76]$, which would explain the accumulation of Pol II observed at the 3 ' end of genes in genome-wide assays $[65,77]$. In spite of these results, the mechanism of termination regulation remains unclear.

\section{Digital responses in single cells}

Live-cell studies are not restricted to studying the kinetic details of initiation, elongation and termination at a single locus; they also have the unique ability to interrogate the timing and robustness of transcription at the level of a cell population or tissue. Such measurements not only yield additional information about transcription mechanisms, but also provide insight into gene expression coordination strategies.

In contrast to ensemble experiments that usually display a graded response to increasing signal levels, microscope snapshots have uncovered digital rules at the single-cell level: in many situations, cells within a population are either highly active or silent. Raising the signal level leads to a gradual increase in the probability for a cell to be 'on', meaning that the smooth response observed at the cell population level results from statistical averaging. Two mechanisms can account for the enrichment in active cells with increased signal: first, all cells might express intermittently, with increasing signal leading to increased burst duration or frequency; alternatively, the signal level could determine the fraction of cells that become active, while the rest of the population remains silent.

Cells have the ability to modulate the characteristics of their transcription bursts to adjust their response to signalling: bacteria have been shown to increase the frequency [21] or the total number of transcription pulses [19] in response to increased signalling. In other words, bacteria behave like digital devices that react to increased stimulus by increasing the number of bursts in a quantized way, rather than increasing the number of transcripts per burst, for example. The two bursting models are compatible with such a signature. In the first one where pulses merely follow upstream signalling changes, the burst modulation would have to be encoded dynamically at a higher level. Indeed, a yeast transcription factor localizes to the nucleus during short, frequencymodulated bursts in response to calcium [78]. Furthermore, during the stress response in bacteria, frequency-modulated bursts of expression are generated by the amplification of stochastic fluctuations through an ultrasensitive phosphoswitch [21]. On the other hand, frequency modulation is not incompatible with spontaneous bursting: one could imagine that a higher concentration of a transcription factor might affect the tendency of a gene to fire bursts. However, there has not yet been any demonstration of such a mechanism. In addition to burst number and frequency modulation, cells can change the shape of transcription bursts: modulation of the burst duration has been invoked to explain the noise signatures of various genes in bacteria [8].

In addition to dynamic pulse regulation, digital responses also involve all-or-nothing cellular states: developmental genes in the slime mould [16,46] and p53 induction in human cells [79] display an all-or-nothing response in which the fraction of pulsing cells increases with increasing signalling (or developmental time), while the pulsing features remain essentially unchanged. Once again, this bimodal response could be merely the propagation of an upstream all-or-nothing signalling cascade, as observed for example with NF-kB nuclear localization [43]. Slow changes in the chromatin coverage and/or marks at the promoter could also account for such responses. Another potential mechanism that could generate all-or-nothing behaviours is the formation of long-lived promoter states through specific contacts between the promoter and distal control sequences [80]. For example, each olfactory sensory neuron in the mouse expresses a single odorant receptor, randomly chosen among around 1,200 genes [81]. The probability of expressing a given gene is modulated by the binding of distal sequences in an all-or-nothing fashion [82]. Single-cell studies of the regulation by distant elements of the $\beta$-globin locus have similarly shown that contacts are rare but stable, resulting in a few 'jackpot cells' within the tissue that express a high level of the target gene [83]. The all-or-nothing control of expression through enhancers might provide a means to switch expression programmes on and off during development: whereas chromatin marks at gene promoters show little change in various differentiated cells, the chromatin signature at enhancers undergoes marked changes [84]. As such, the nature of the enhancer marks before differentiation can predict the subsequent fate of the associated gene, suggesting that chromatin modifications modulate enhancer contact frequency, rather than vice versa [85]. 


\section{Choosing a gene expression strategy}

The ongoing progress in resolution of imaging studies is revealing a wealth of transcription regulation modes at the single-molecule level: some genes are constitutively 'on', while others only transcribe through short bursts. Cells modulate the shape and frequency of the bursts to respond to environmental changes. Why some genes have adopted one mode of expression rather than another is still unclear. However, some broad principles seem to emerge: in yeast, signal response genes are mostly 'bursty', whereas housekeeping genes tend to have a more constitutive expression, consistent with their promoter chromatin architecture (Fig 2). Digital pulses have certain advantages over analogue expression during signal response. First, the cell can assess the efficiency of the response between each pulse and make a decision to generate another pulse if necessary. The cell can tune the duration of the response to the actual needs of the cell. Second, pulses of signalling allow one factor to maintain a constant ratio between the expression levels of multiple target genes over the whole range of responses [78]. Finally, bursting generates expression variability within an isogenic cell population, which increases the chances of survival upon abrupt environmental changes. Importantly, cellular responses have the potential to incorporate both digital and analogue regulatory levers to fine-tune their response [43]. At the other end of the dynamic spectrum, constitutive stochastic expression can provide a 'low-cost' solution to robustly expressing a housekeeping gene: the gene is constantly open for business, and generates mRNAs every time a transcription factor binds to the promoter [13]. Provided the lifetime of the mRNA and/or the downstream protein is long enough, the randomness of mRNA synthesis gets averaged out over time, yielding a robust expression $[34,38]$. As a consequence, uncorrelated transcription of functionally related genes can still yield robust downstream expression profiles, allowing the cell to reliably build multiprotein complexes [36]. The lifetime of the gene expression products - mRNA and associated protein — can therefore act as a buffer for the fluctuations in their synthesis [86]. This simple result also bears consequences for pulsed transcription: bursts will result in temporal phenotype variations only if the lifetime of the gene expression products is similar or shorter than the typical period of a burst.

\section{Open questions}

Techniques that monitor transcription by single genes in live cells are instrumental in our understanding of the dynamics of gene expression. It is now clear that gene networks, architecture, transcription factor mobility, genome structure and molecular details of interactions at the promoter convolve to create precise temporal profiles of gene expression [39]. However, many questions remain unanswered (see Sidebar A). The molecular details of spontaneous bursting are unknown. How it can occur both in the repressive chromatin environment found in eukaryotes and also in the more broadly expressed prokaryotic genome is unclear. In parallel with improvements in our ability to resolve transcription events in single cells by microscopy, progress in mapping chromatin and factor binding over entire genomes is helping to refine our understanding of the landscape of gene expression regulation: transcription factors bind broadly across the genome [87]; chromatin structure is more nuanced than traditionally thought $[88,89]$; transcription can occur in both directions from the promoter [65,90]; enhancer regions are widespread across the genome [84]. An integrated model that can predict how all these elements direct the transcriptional output of a gene in response to a given signal is the ultimate goal.

\section{Sidebar A | In need of answers}

(i) What is (are) the biological origin(s) of transcriptional bursting?

(ii) How can short (minutes or less) interactions of the transcription machinery components at the gene locus generate sustained (hours plus) expression profiles?

(iii) Does the gene locus undergo conformation changes? How do they influence transcription kinetics and efficiency?

(iv) Is bursting regulation different in prokaryotes compared with eukaryotes? Do lower eukaryotes having a compact genome (such as yeast) display a bursting regulation distinct from higher eukaryotes?

(v) What impact do the promoter sequence and chromatin structure have on transcriptional noise?

\section{ACKNOWLEDGEMENTS}

We thank D. R. Larson for sharing analysis software, D. Suter and N. Molina for communicating data, and J. R. Chubb for sharing unpublished data and discussion. This work was supported by National Institutes of Health grants GM84364, GM57071 and GM86217.

\section{CONFLICT OF INTEREST}

The authors declare that they have no conflict of interest.

\section{REFERENCES}

1. Balazsi G, van Oudenaarden A, Collins JJ (2011) Cellular decision making and biological noise: from microbes to mammals. Cell 144: 910-925

2. Darzacq X et al (2009) Imaging transcription in living cells. Annu Rev Biophys 38: 173-196

3. Locke JC, Elowitz MB (2009) Using movies to analyse gene circuit dynamics in single cells. Nat Rev Microbio/ 7: 383-392

4. Chubb JR, Liverpool TB (2010) Bursts and pulses: insights from single cell studies into transcriptional mechanisms. Curr Opin Genet Dev 20: 478-484

5. Femino AM, Fay FS, Fogarty K, Singer RH (1998) Visualization of single RNA transcripts in situ. Science 280: 585-590

6. Raj A, Peskin CS, Tranchina D, Vargas DY, Tyagi S (2006) Stochastic mRNA synthesis in mammalian cells. PLoS Biol 4: e309

7. Raj A, Rifkin SA, Andersen E, van Oudenaarden A (2010) Variability in gene expression underlies incomplete penetrance. Nature 463: 913-918

8. So LH, Ghosh A, Zong C, Sepulveda LA, Segev R, Golding I (2011) General properties of transcriptional time series in Escherichia coli. Nat Genet 43: 554-560

9. Yunger S, Rosenfeld L, Garini Y, Shav-TalY (2010) Single-allele analysis of transcription kinetics in living mammalian cells. Nat Methods 7: 631-633

10. Zenklusen D, Larson DR, Singer RH (2008) Single-RNA counting reveals alternative modes of gene expression in yeast. Nat Struct Mol Biol 15: 1263-1271

11. Bertrand E, Chartrand P, Schaefer M, Shenoy SM, Singer RH, Long RM (1998) Localization of ASH1 mRNA particles in living yeast. Mol Cell 2: 437-445

12. Lionnet T, Czaplinski K, Darzacq X, Shav-Tal Y, Wells AL, Chao JA, Park HY, de Turris V, Lopez-Jones M, Singer RH (2011) A transgenic mouse for in vivo detection of endogenous labeled mRNA. Nat Methods 8: 165-170

13. Larson DR, Zenklusen D, Wu B, Chao JA, Singer RH (2011) Real-time observation of transcription initiation and elongation on an endogenous yeast gene. Science 332: $475-478$

14. Darzacq X, Shav-Tal Y, de Turris V, Brody Y, Shenoy SM, Phair RD, Singer RH (2007) In vivo dynamics of RNA polymerase II transcription. Nat Struct Mol Biol 14: 796-806

15. Janicki SM et al (2004) From silencing to gene expression: real-time analysis in single cells. Cell 116: 683-698

16. Chubb JR, Trcek T, Shenoy SM, Singer RH (2006) Transcriptional pulsing of a developmental gene. Curr Biol 16: 1018-1025

17. Golding I, Paulsson J, Zawilski SM, Cox EC (2005) Real-time kinetics of gene activity in individual bacteria. Cell 123: 1025-1036 
18. Tyagi S (2009) Imaging intracellular RNA distribution and dynamics in living cells. Nat Methods 6: $331-338$

19. Friedman N, Vardi S, Ronen M, Alon U, Stavans J (2005) Precise temporal modulation in the response of the SOS DNA repair network in individual bacteria. PLoS Bio/ 3: e238

20. Lahav G, Rosenfeld N, Sigal A, Geva-Zatorsky N, Levine AJ, Elowitz MB, Alon U (2004) Dynamics of the p53-Mdm2 feedback loop in individual cells. Nat Genet 36: 147-150

21. Locke JC, Young JW, Fontes M, Hernandez Jimenez MJ, Elowitz MB (2011) Stochastic pulse regulation in bacterial stress response. Science 334: 366-369

22. Cai L, Friedman N, Xie XS (2006) Stochastic protein expression in individual cells at the single molecule level. Nature 440: 358-362

23. Yu J, Xiao J, Ren X, Lao K, Xie XS (2006) Probing gene expression in live cells, one protein molecule at a time. Science 311: 1600-1603

24. Harper CV et al (2011) Dynamic analysis of stochastic transcription cycles. PLoS Bio/ 9: e1000607

25. Suter DM, Molina N, Gatfield D, Schneider K, Schibler U, Naef F (2011) Mammalian genes are transcribed with widely different bursting kinetics. Science 332: 472-474

26. Blake WJ, Kaern M, Cantor CR, Collins JJ (2003) Noise in eukaryotic gene expression. Nature 422: 633-637

27. Pare A, Lemons D, Kosman D, Beaver W, Freund Y, McGinnis W (2009) Visualization of individual Scr mRNAs during Drosophila embryogenesis yields evidence for transcriptional bursting. Curr Biol 19: 2037-2042

28. Raj A, van den Bogaard P, Rifkin SA, van Oudenaarden A, Tyagi S (2008) Imaging individual mRNA molecules using multiple singly labeled probes. Nat Methods 5: 877-879

29. Newlands S, Levitt LK, Robinson CS, Karpf AB, Hodgson VR, Wade RP, Hardeman EC (1998) Transcription occurs in pulses in muscle fibers. Genes Dev 12: 2748-2758

30. Gorski SA, Snyder SK, John S, Grummt I, Misteli T (2008) Modulation of RNA polymerase assembly dynamics in transcriptional regulation. Mol Cell 30: 486-497

31. Tan RZ, van Oudenaarden A (2010) Transcript counting in single cells reveals dynamics of rDNA transcription. Mol Syst Bio/ 6: 358

32. Bar-Even A, Paulsson J, Maheshri N, Carmi M, O'Shea E, Pilpel Y, Barkai N (2006) Noise in protein expression scales with natural protein abundance. Nat Genet 38: 636-643

33. Karpova TS, Kim MJ, Spriet C, Nalley K, Stasevich TJ, Kherrouche Z, Heliot L, McNally JG (2008) Concurrent fast and slow cycling of a transcriptional activator at an endogenous promoter. Science 319: 466-469

34. Taniguchi Y, Choi PJ, Li GW, Chen H, Babu M, Hearn J, Emili A, Xie XS (2010) Quantifying E coli proteome and transcriptome with singlemolecule sensitivity in single cells. Science 329: 533-538

35. Lionnet T, Wu B, Grunwald D, Singer RH, Larson DR (2011) Nuclear physics: quantitative single-cell approaches to nuclear organization and gene expression. Cold Spring Harbor Symp Quant Biol 75: 113-126

36. Gandhi SJ, Zenklusen D, Lionnet T, Singer RH (2011) Transcription of functionally related constitutive genes is not coordinated. Nat Struct Mol Biol 18: 27-34

37. Garcia HG, Sanchez A, Kuhlman T, Kondev J, Phillips R (2010) Transcription by the numbers redux: experiments and calculations that surprise. Trends Cell Biol 20: 723-733

38. Paulsson J (2005) Models of stochastic gene expression. Phys Life Rev 2: 157-175

39. Yosef N, Regev A (2011) Impulse control: temporal dynamics in gene transcription. Cell 144: 886-896

40. Elowitz MB, Leibler S (2000) A synthetic oscillatory network of transcriptional regulators. Nature 403: 335-338

41. Hilfinger A, Paulsson J (2011) Separating intrinsic from extrinsic fluctuations in dynamic biological systems. Proc Natl Acad Sci USA 108: $12167-12172$

42. Goh KI, Barabasi A (2008) Burstiness and memory in complex systems. Europhysics Lett 81: 48002

43. Tay S, Hughey JJ, Lee TK, Lipniacki T, Quake SR, Covert MW (2010) Single-cell NF-kB dynamics reveal digital activation and analogue information processing. Nature 466: $267-271$

44. Larson DR, Singer RH, Zenklusen D (2009) A single molecule view of gene expression. Trends Cell Biol 19: 630-637
45. Huh D, Paulsson J (2011) Non-genetic heterogeneity from stochastic partitioning at cell division. Nat Genet 43: 95-100

46. Stevense M, Muramoto T, Muller I, Chubb JR (2010) Digital nature of the immediate-early transcriptional response. Development 137: 579-584

47. Lemon B, Tjian R (2000) Orchestrated response: a symphony of transcription factors for gene control. Genes Dev 14: 2551-2569

48. Sikorski TW, Buratowski S (2009) The basal initiation machinery: beyond the general transcription factors. Curr Opin Cell Biol 21: 344-351

49. Stasevich TJ, McNally JG (2011) Assembly of the transcription machinery: ordered and stable, random and dynamic, or both? Chromosoma 120: 533-545

50. Raser JM, O'Shea EK (2004) Control of stochasticity in eukaryotic gene expression. Science 304: 1811-1814

51. Jiang C, Pugh BF (2009) Nucleosome positioning and gene regulation: advances through genomics. Nat Rev Genet 10: 161-172

52. Field Y, Kaplan N, Fondufe-Mittendorf Y, Moore IK, Sharon E, Lubling Y, Widom J, Segal E (2008) Distinct modes of regulation by chromatin encoded through nucleosome positioning signals. PLoS Comput Bio/ 4: e1000216

53. Tirosh I, Barkai N (2008) Two strategies for gene regulation by promoter nucleosomes. Genome Res 18: 1084-1091

54. Cairns BR (2009) The logic of chromatin architecture and remodelling at promoters. Nature 461: 193-198

55. Bai L, Charvin G, Siggia ED, Cross FR (2010) Nucleosome-depleted regions in cell-cycle-regulated promoters ensure reliable gene expression in every cell cycle. Dev Cell 18: 544-555

56. Lam FH, Steger DJ, O'Shea EK (2008) Chromatin decouples promoter threshold from dynamic range. Nature 453: 246-250

57. Zaugg JB, Luscombe NM (2012) A genomic model of condition-specific nucleosome behavior explains transcriptional activity in yeast. Genome Res 22: 84-94

58. Hager GL, McNally JG, Misteli T (2009) Transcription dynamics. Mol Cell 35: 741-753

59. Larson DR (2011) What do expression dynamics tell us about the mechanism of transcription? Curr Opin Genet Dev 21: 591-599

60. Metivier R, Penot G, Hubner MR, Reid G, Brand H, Kos M, Gannon F (2003) Estrogen receptor- $\alpha$ directs ordered, cyclical, and combinatorial recruitment of cofactors on a natural target promoter. Cell 115: 751-763

61. Muramoto T, Muller I, Thomas G, Melvin A, Chubb JR (2010) Methylation of $\mathrm{H} 3 \mathrm{~K} 4$ is required for inheritance of active transcriptional states. Curr Biol 20: 397-406

62. Brickner JH (2010) Transcriptional memory: staying in the loop. Curr Biol 20: R20-R21

63. Yao J, Munson KM, Webb WW, Lis JT (2006) Dynamics of heat shock factor association with native gene loci in living cells. Nature 442: 1050-1053

64. Sutherland H, Bickmore WA (2009) Transcription factories: gene expression in unions? Nat Rev Genet 10: 457-466

65. Core LJ, Waterfall JJ, Lis JT (2008) Nascent RNA sequencing reveals widespread pausing and divergent initiation at human promoters. Science 322: 1845-1848

66. Muse GW, Gilchrist DA, Nechaev S, Shah R, Parker JS, Grissom SF, Zeitlinger J, Adelman K (2007) RNA polymerase is poised for activation across the genome. Nat Genet 39: 1507-1511

67. Gilchrist DA, Dos Santos G, Fargo DC, Xie B, Gao Y, Li L, Adelman K (2010) Pausing of RNA polymerase II disrupts DNA-specified nucleosome organization to enable precise gene regulation. Cell 143: 540-551

68. Boettiger AN, Levine M (2009) Synchronous and stochastic patterns of gene activation in the Drosophila embryo. Science 325: 471-473

69. Ghosh SK, Missra A, Gilmour DS (2011) Negative elongation factor accelerates the rate at which heat shock genes are shut off by facilitating dissociation of heat shock factor. Mol Cell Biol 31: 4232-4243

70. Chopra VS, Cande J, Hong JW, Levine M (2009) Stalled Hox promoters as chromosomal boundaries. Genes Dev 23: 1505-1509

71. Mueller F, Mazza D, Stasevich TJ, McNally JG (2010) FRAP and kinetic modeling in the analysis of nuclear protein dynamics: what do we really know? Curr Opin Cell Biol 22: 403-411

72. Maiuri P, Knezevich A, De Marco A, Mazza D, Kula A, McNally JG, Marcello A (2011) Fast transcription rates of RNA polymerase II in human cells. EMBO Rep 12: 1280-1285 
73. Dobrzynski M, Bruggeman FJ (2009) Elongation dynamics shape bursty transcription and translation. Proc Natl Acad Sci USA 106: 2583-2588

74. Mason PB, Struhl K (2005) Distinction and relationship between elongation rate and processivity of RNA polymerase II in vivo. Mol Cell 17: $831-840$

75. de la Mata M, Alonso CR, Kadener S, Fededa JP, Blaustein M, Pelisch F, Cramer P, Bentley D, Kornblihtt AR (2003) A slow RNA polymerase II affects alternative splicing in vivo. Mol Cell 12: 525-532

76. Boireau S, Maiuri P, Basyuk E, de la Mata M, Knezevich A, PradetBalade B, Backer V, Kornblihtt A, Marcello A, Bertrand E (2007) The transcriptional cycle of HIV-1 in real-time and live cells. J Cell Biol 179: 291-304

77. Lian Z, Karpikov A, Lian J, Mahajan MC, Hartman S, Gerstein M, Snyder M, Weissman SM (2008) A genomic analysis of RNA polymerase II modification and chromatin architecture related to 3' end RNA polyadenylation. Genome Res 18: 1224-1237

78. Cai L, Dalal CK, Elowitz MB (2008) Frequency-modulated nuclear localization bursts coordinate gene regulation. Nature 455: 485-490

79. Geva-Zatorsky N et al (2006) Oscillations and variability in the p53 system. Mol Syst Biol 2: 20060033

80. Walters MC, Fiering S, Eidemiller J, Magis W, Groudine M, Martin DI (1995) Enhancers increase the probability but not the level of gene expression. Proc Natl Acad Sci USA 92: 7125-7129

81. Lomvardas S, Barnea G, Pisapia DJ, Mendelsohn M, Kirkland J, Axel R (2006) Interchromosomal interactions and olfactory receptor choice. Cell 126: 403-413

82. Khan $M$, Vaes $E$, Mombaerts $P$ (2011) Regulation of the probability of mouse odorant receptor gene choice. Cell 147: 907-921

83. Noordermeer D, de Wit E, Klous $\mathrm{P}$, van de Werken $\mathrm{H}$, Simonis $\mathrm{M}$ Lopez-Jones M, Eussen B, de Klein A, Singer RH, de Laat W (2011) Variegated gene expression caused by cell-specific long-range DNA interactions. Nat Cell Biol 13: 944-951

84. Heintzman ND et al (2009) Histone modifications at human enhancers reflect global cell-type-specific gene expression. Nature 459: 108-112

85. Cui K, Zang C, Roh TY, Schones DE, Childs RW, Peng W, Zhao K (2009) Chromatin signatures in multipotent human hematopoietic stem cells indicate the fate of bivalent genes during differentiation. Cell Stem Cell 4: 80-93

86. Eldar A, Elowitz MB (2010) Functional roles for noise in genetic circuits Nature 467: 167-173

87. MacQuarrie KL, Fong AP, Morse RH, Tapscott SJ (2011) Genome-wide transcription factor binding: beyond direct target regulation. Trends Genet 27: 141-148

88. Ernst J, Kellis M (2010) Discovery and characterization of chromatin states for systematic annotation of the human genome. Nat Biotechnol 28: $817-825$

89. Filion GJ et al (2010) Systematic protein location mapping reveals five principal chromatin types in Drosophila cells. Cell 143: 212-224

90. Churchman LS, Weissman JS (2011) Nascent transcript sequencing visualizes transcription at nucleotide resolution. Nature 469: 368-373

91. Lee W, Tillo D, Bray N, Morse RH, Davis RW, Hughes TR, Nislow C (2007) A high-resolution atlas of nucleosome occupancy in yeast. Nat Genet 39: 1235-1244

92. Stevense M, Muramoto T, Müller I, Chubb JR (2010) Digital nature of the immediate-early transcriptional response. Development 137: 579-584

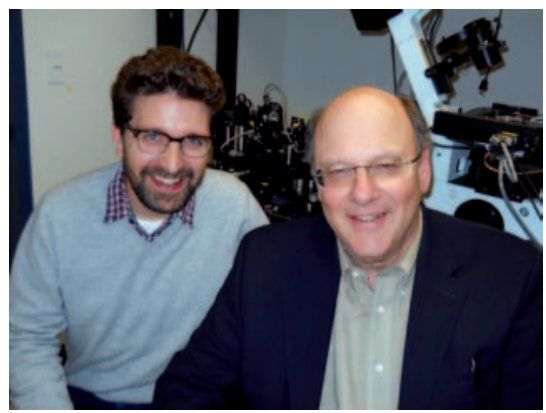

Timothée Lionnet \& Robert H. Singer 